\title{
GENERACIÓN EFICIENTE DE MOSAICOS PARA INSPECCIÓN DE INFRAESTRUCTURAS LINEALES MEDIANTE ROBOTS AÉREOS
}

\author{
R. Tapia, J.R. Martinez-de Dios, A. Ollero \\ Robotics Vision and Control Laboratory, Universidad de Sevilla, Escuela Superior de Ingenieros \\ c/Camino de los Descubrimientos s/n, 41092 Seville, Spain; rautaplop@alum.us.es, jdedios@us.es, aollero@us.es
}

\begin{abstract}
Resumen
Este artículo presenta un método de generación de mosaicos mediante imágenes tomadas por robots aéreos para aplicaciones de inspección de infraestructuras lineales. El método ha sido diseñado utilizando las hipótesis del problema para reducir su coste computacional, manteniendo su comportamiento preciso y robusto. En particular, utiliza la hipótesis de vuelo rectilíneo y la estimación del desplazamiento entre imágenes consecutivas para seleccionar zonas de interés evitando detectar y asociar características en toda la imagen, lo que además reduce los valores atípicos y, por tanto, simplifica la optimización para el cálculo de la transformación entre imágenes. El método ha sido validado experimentalmente en misiones de inspección de oleoductos mediante robots aéreos.
\end{abstract}

Palabras clave: Robótica aérea, generación de mosaicos, inspección

\section{INTRODUCCIÓN}

En los últimos años se ha multiplicado la investigación en el empleo de sistemas aéreos no tripulados (UAS) para aplicaciones de inspección y mantenimiento. Los UAS permiten inspecciones más flexibles, ágiles, seguras y rápidas sin necesidad de desplegar infraestructuras, evitan la inspección manual en ambientes de alta peligrosidad y reducen de forma muy relevante el número de horas de personal necesarias para realizar la inspección, generando reducciones drásticas de costes. Los proyectos H2020 AEROARMS [1] o H2020 AEROBI [2] son dos ejemplos de esfuerzos de $\mathrm{I}+\mathrm{D}$ desarrollados en el Laboratorio de Robótica, Visión y Control de la Universidad de Sevilla dedicados a la inspección de instalaciones industriales y de puentes, respectivamente.

Existe un gran número de infraestructuras e instalaciones que tienen una naturaleza lineal y se extienden durante cientos de metros o kilómetros de distancia. La inspección de gasoductos, oleoductos y tendido eléctrico son tres ejemplos. La inspección de infraestructuras lineales requiere la generación de mosaicos visuales de gran tamaño. Además, el empleo operacional de estos métodos en la organización productiva de las empresas requiere su funcionamiento en condiciones cercanas al tiempo real. Aunque se han desarrollado un alto número de métodos de generación de mosaicos en los últimos años [7] [20], la gran mayoría no está diseñada para una aplicación concreta y en general tienen un coste computacional muy alto, lo que dificulta su implementación.

Este artículo presenta un método de generación de mosaicos mediante imágenes tomadas por robots aéreos para aplicaciones de inspección de infraestructuras lineales. El método ha sido diseñado para tener un comportamiento robusto y preciso con un moderado coste computacional. En particular, el método estima la odometría del robot para predecir las zonas de la imagen que se solapan en imágenes consecutivas y evitar el procesamiento de las imágenes completas. La asociación de características se realiza utilizando ventanas calculadas a partir de la odometría del robot y de las medidas de GPS. Además, emplea características ORB [19] y realiza el cálculo de la transformación mediante optimización por descomposición SVD, lo que le confiere notable robustez y eficiencia.

La estructura del resto del artículo es como sigue. La Sección 2 resume el estado del arte en las técnicas de generación de mosaicos. El esquema general del método se presenta en la Sección 3. Las Secciones 4, 5 y 6 presentan las tres fases del método: extracción de características, asociación de características y cálculo de la transformación homogénea. La validación experimental en misiones de inspección de oleoductos se presenta en la Sección 7. La Sección 8 resume las conclusiones y los desarrollos futuros.

\section{ESTADO DEL ARTE}

La generación de mosaicos es una técnica de procesamiento de imagen que permite combinar varias imágenes digitales para formar una de mayor tamaño. Se trata de un caso especial de corrección geométrica en el que una imagen se ajusta a otra con la que se relaciona mediante una transforma- 
ción homogénea [9]. Las técnicas de generación de mosaicos son un campo de investigación activo debido a su amplio rango de aplicaciones [3] [15].

Se pueden diferenciar dos grandes categorías de técnicas de generación de mosaicos: las que no involucran extracción de características -conocidas como métodos directos- y las técnicas con extracción de características, denominadas métodos no directos.

Dentro de las técnicas sin extracción de características, predominan aquellas en las que el mosaico es generado a partir de los parámetros de posición, estimados mediante la minimización del error cuadrático de la diferencia de intensidades en las zonas donde las imágenes deben superponerse. En [10] se describe la técnica de minimización más usada por estos métodos, basada en la optimización de Levenberg-Marquardt. La calidad del mosaico resultante depende directamente del resultado de la minimización, siendo en ocasiones un gran problema su sensibilidad ante mínimos locales, como se pone de manifiesto en [11]. También se incluyen las técnicas en las que las imágenes que forman el mosaico se reconstruyen sobre un cilindro [18], una esfera o cualquier otra figura de revolución [5] para conformar un entorno tridimensional. Este tipo de técnicas no son adecuadas para inspecciones de grandes superficies por su necesidad de un gran número de puntos en común y por sus elevados tiempos de computación.

Los métodos basados en características suelen comprender tres etapas: 1) extracción de características, 2) asociación de características y 3) cálculo de la homografía que relaciona las imágenes. Existen distintos tipos de características, siendo los más empleados los puntos, los bordes y las regiones. Entre los extractores más utilizados actualmente en generación de mosaicos se encuentran los algoritmos SIFT, SURF y ORB, [12].

En la literatura asociada a la generación de mosaicos se pueden encontrar métodos de carácter muy general [6]. En [17] se detalla como generar distintas secuencias de ajuste de las imágenes mediante variadas transformaciones. Sin embargo, es difícil encontrar métodos centrados en como ganar eficiencia computacional mediante la particularización a casos concretos. Uno de los puntos a destacar del método presentado en este artículo es el uso de las medidas GPS para obtener ciertas estimaciones que permitan reducir el tiempo de generación del mosaico.

Los algoritmos basados en características suelen clasificarse en función del tipo de transformación que pueden efectuar a las imágenes. La diferencia entre cada categoría reside en la complejidad de los algoritmos y en su tiempo de ejecución. Para los experimentos realizados, se ha optado por tener en cuenta los grados de libertad mínimos para conseguir eficiencia computacional.

\section{ESQUEMA GENERAL DEL MÉTODO}

Se desea realizar el mosaico de un escenario lineal y aproximadamente plano mediante un robot aéreo dotado de una cámara, GPS, IMU y altímetro. Además, el robot está equipado con sensores relacionados con el tipo de inspección que realizará. En nuestro caso -inspección de gasoductosestá equipado con un sensor para medir la concentración en aire de un cierto gas. El método tiene como objetivo generar un mosaico a partir de las imágenes e introducir en él cierta información de utilidad al operario, en concreto, referencias de posición GPS y datos de concentraciones de gases. Se desea que la generación del mosaico sea realizado en un tiempo cercano al tiempo real y bastante inferior al de muchas otras técnicas de generación de mosaico actualmente existentes. El algoritmo deberá operar con un número a priori desconocido de imágenes, de forma que se permita afrontar inspecciones de terrenos de gran extensión.

El algoritmo desarrollado se compone de tres módulos. Los métodos y algoritmos elegidos en cada uno de ellos han sido diseñado con dos premisas: eficiencia computacional y escalabilidad. El primer módulo se encarga de la extracción de características de las imágenes capturadas haciendo uso de ORB [19], uno de los algoritmos más rápidos actualmente utilizados. Se extraen características en las regiones de las imágenes que se superponen entre dos imágenes consecutivas, lo que consigue reducir el cómputo.

El segundo módulo obtiene correlaciones entre los puntos extraídos. Emplea ventanas calculadas a partir de las medidas GPS para mejorar su eficiencia computacional. En el tercer módulo se calcula la transformación homogénea que relaciona los puntos previamente relacionados. Se emplea un método basado en la descomposición en valores singulares (SVD) para reducir el coste computacional de esta optimización no lineal.

El esquema propuesto emplea el hecho de que el vuelo tendrá una gran componente longitudinal para realizar simplificaciones que permitan reducir el coste computacional y mejorar la robustez. El método supone que el vuelo se realizará con altura elevada y aproximadamente constante y con variaciones en cabeceo y alabeo casi nulas. No obstante, puede ser fácilmente extrapolable a otros casos de carácter más general. 


\section{EXTRACCIÓN DE CARACTERÍSTICAS}

\subsection{Detección de características}

El primer paso es la detección de puntos de interés. ORB [19] es un detector de características visuales que combina los algoritmos FAST y BRIEF. Se ha elegido ORB por su mayor eficiencia computacional frente a alternativas como SIFT [16] y SURF [4]. Otra de las ventajas de ORB frente a otros extractores es su invarianza respecto a la rotación, escalado y perspectiva, lo cual será utilidad para relacionar dos imágenes consecutivas cuando exista una variación de la guiñada o de la altura, o para dotarle de estabilidad frente a pequeñas variaciones de cabeceo y alabeo.

Uno de los parámetros más importante del algoritmo ORB es el número máximo de puntos característicos a encontrar, el cual tiene implicaciones en el coste computacional tanto en extracción como, sobre todo, en la asociación de características. El edge threshold, o umbral de borde, es otro de los parámetros de importancia. Se trata del tamaño del marco de la imagen donde no habrá extracción de características. Este umbral permite evitar posibles problemas asociados a la extracción de puntos en zonas cercanas a los límites de la imagen. Otro motivo por el que podría preferirse no buscar puntos de interés en entornos próximos a los límites de la imagen es evitar las distorsiones ópticas de la lente, que suelen ser mayores en los bordes de la imagen. Para los experimentos realizados se han extraído 100 características en cada imagen, 50 en la zona izquierda y 50 en la derecha, y se ha fijado un umbral de borde de 50 píxeles.

Para mejorar la eficiencia computacional se ha evitado la extracción de características en puntos no comunes a dos imágenes consecutivas. Los puntos no comunes han sido calculados a partir de una estimación del desplazamiento, obtenida con las coordenadas GPS y altitud del robot asociadas a cada captura. En la Figura 1 puede verse un ejemplo de extracción de características de una imagen tomada en los experimentos. Se muestran los puntos característicos únicamente en la zona común con la imagen inmediatamente anterior, evitando los bordes de la imagen. Las zonas no comunes se muestran en tonos más oscuros.

\subsection{Extracción de descriptores}

Una vez detectados los puntos de interés, se extraen las características. Un extractor genera un vector de datos que describe la vecindad entorno a cada uno de los puntos de interés detectados. Cada punto de interés tendrá asociado un identifi- cador. Idealmente, los descriptores son invariantes ante transformaciones, lo que permite identificar la misma característica cuando la imagen haya sido transformada.

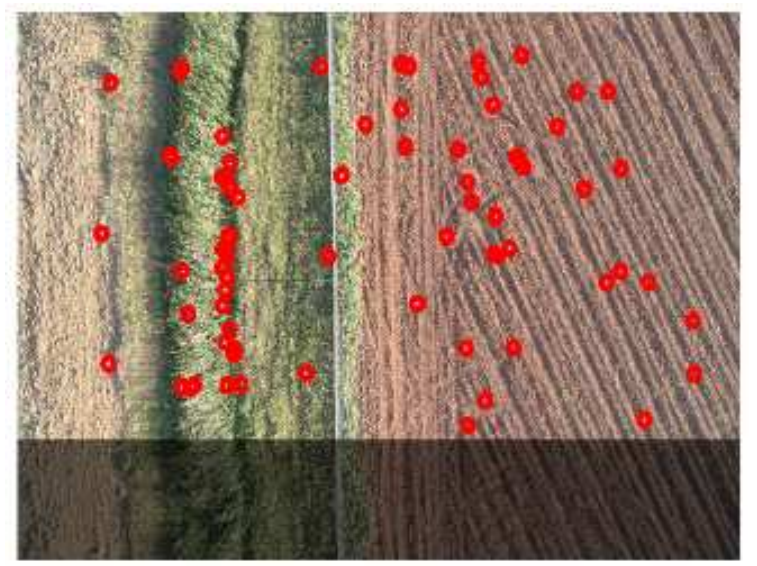

Figura 1: Extracción de características en una imagen tomada en los experimentos de inspección de gasoductos.

ORB utiliza un vector de valores binarios con un tamaño de 256 bits. Sea $d\left(p_{1}, p_{2}\right)$ una componente del vector de descripción del característica $f, S$ una sección de la imagen y $S(p)$ la intensidad de $S$ en el punto $p=(x, y)^{T}$ :

$$
d\left(p_{1}, p_{2}\right):=\left\{\begin{array}{lll}
1 & \text { si } & S\left(p_{1}\right)<S\left(p_{2}\right) \\
0 & \text { si } & S\left(p_{1}\right) \geq S\left(p_{2}\right)
\end{array}\right.
$$

El vector de descripción de $f$ vendrá dado por:

$$
D_{f}=\sum_{1 \leq k \leq 256} 2^{k-1} d\left(a_{k}, b_{k}\right),
$$

donde los valores $a_{k}$ y $b_{k}$ se escogen según una distribución Gaussiana en torno al centro de $S$.

\section{ASOCIACIÓN DE CARACTERÍSTICAS}

Una vez que en imágenes consecutivas se han detectado características y se han obtenido sus descriptores, el siguiente paso consiste en asociar las características de ambas imágenes. Muchos métodos realizan la asociación empleando técnicas de correlación por fuerza bruta, consistente en comparar los descriptores de todos los puntos característicos de una imagen con todos los de la otra imagen [14]. Sin embargo, la correlación por fuerza bruta requiere un alto coste computacional, sobre todo para relacionar un gran número de puntos. En nuestro método se ha optado por implementar un método más eficiente, que obtiene la correlación mediante el cálculo de la distancia de Hamming de los descriptores de los puntos de interés 
y que tiene en cuenta la estimación del desplazamiento del robot aéreo.

\subsection{Comparación de características}

Se conoce como correlación de características al proceso mediante el cual se relacionan entre sí los puntos de interés de dos imágenes. El descriptor usado por ORB es un descriptor binario, lo que permite obtener métricas de parecido entre características de forma muy eficiente, empleando la distancia de Hamming. Se define la distancia de Hamming entre la cadena $a$ y la cadena $b$ como el número de bits que se deben cambiar en $a$ para transformarla en $b$. Esta distancia puede ser calculada utilizando la disyunción exclusiva (XOR):

$$
d(a, b)=\text { bit_count }(a \oplus b),
$$

donde:

$$
a \oplus b=(a \cup b) \backslash(a \cap b)=(a \cup b) \cap \overline{(a \cap b)}
$$

El hecho de poder calcular la distancia de Hamming de dos descriptores con esta operación es una gran ventaja en cuanto a coste computacional, ya que XOR es una de las operaciones más rápidas que un procesador puede llevar a cabo. Actualmente todas las CPUs soportan esta operación y son capaces de ejecutarla en menos de un ciclo de reloj. Además, muchos procesadores poseen la capacidad de ejecutar varios XOR en paralelo.

Una vez obtenida la distancia entre los descriptores de dos características, es necesario determinar si esa unión debe ser considerada como buena. Para ello, se escogerán las $n$ que presenten la menor distancia. Esta forma de proceder hace innecesario un tratamiento de posibles valores atípicos, evitándose todo el coste computacional requerido por algoritmos como RANSAC.

\subsection{Ventanas de comparación}

La correlación de los puntos característicos puede hallarse comparando todos descriptores de las dos imágenes. Sin embargo, el coste computacional que se requiere es relativamente elevado. Para reducir el tiempo de ejecución se seleccionan ventanas en las imágenes, que reducirán el número de comparaciones a realizar. El cálculo de estas ventanas se realiza con la estimación de la posición del robot, ofrecida por el GPS y por la medida de la guiñada.

Sea $\Delta x=x_{k}-x_{k-1}$ e $\Delta y=y_{k}-y_{k-1}$ obtenidas a partir de las posiciones $\left(x_{k}, y_{k}\right)$ y $\left(x_{k-1}, y_{k-1}\right)$, el desplazamiento en línea recta efectuado por el robot es:

$$
d=\sqrt{\Delta x^{2}+\Delta y^{2}}
$$

Conocida la medida de la guinada respecto a una referencia, $\theta$, y conocida la posición de un punto en la imagen $I_{k},\left(x_{1}, y_{1}\right)$, puede estimarse la posición que ocupó ese punto en la imagen $I_{k-1}$ como:

$$
\left(x_{0}, y_{0}\right) \approx\left(x_{1}-d \sin \theta, y_{1}-d \cos \theta\right)
$$

Las ventanas son seleccionadas lo suficientemente amplias como para absorber el error en las medidas de posición. En los experimentos realizados se han utilizado ventanas de un tamaño aproximadamente igual al $15 \%$ de la altura de la imagen.

En la Figura 2 puede verse un ejemplo de asociación de características de dos imágenes tomadas en los experimentos. Se muestran los puntos característicos emparejados del mismo color, mientras que aquellos con una distancia superior al umbral aparecen en negro. Las cruces de las imágenes son puntos tomados como referencias. La cruz roja de la imagen inferior ha sido obtenida a partir de la estimación detallada en esta sección. El marco rojo es un ejemplo del ventana.

\section{CÁlCULO DE LA TRANSFORMACIÓN HOMOGÉNEA}

El último paso consiste en encontrar la transformación que relaciona los conjuntos de puntos asociados, calculando para ello los parámetros que proporcionen el mejor ajuste del modelo mediante la minimización del error cuadrático.

Un punto $P=(x, y)^{T}$ puede ser expresado en coordenadas homogéneas como $P=(s x, s y, s)^{T}$, donde la nueva coordenada representa un factor de escala. En lo sucesivo, se supondrá $s=1$ sin perder generalidad. Empleando coordenadas homogéneas, la transformación entre dos puntos viene dada por $x_{2}=T x_{1}$, donde $T$ es la matriz de transformación. Las condiciones de vuelo permiten aproximar dicha matriz de la forma:

$$
T=\left[\begin{array}{ccc}
\cos \theta & -\sin \theta & p_{x} \\
\sin \theta & \cos \theta & p_{y} \\
0 & 0 & 1
\end{array}\right]
$$

Esta transformación tiene como parámetros la traslación $\left(p_{x}, p_{y}\right)$ y la orientación $\theta$, y supone una variación en los ángulos de cabeceo y alabeo nula entre dos imágenes consecutivas. Esta particularización implica una disminución considerable del tiempo de cómputo, por lo que el algoritmo será capaz de trabajar con conjuntos grandes de puntos en un tiempo relativamente pequeño.

La expresión $x_{2} \approx T x_{1}$, puede ser reescrita de la 

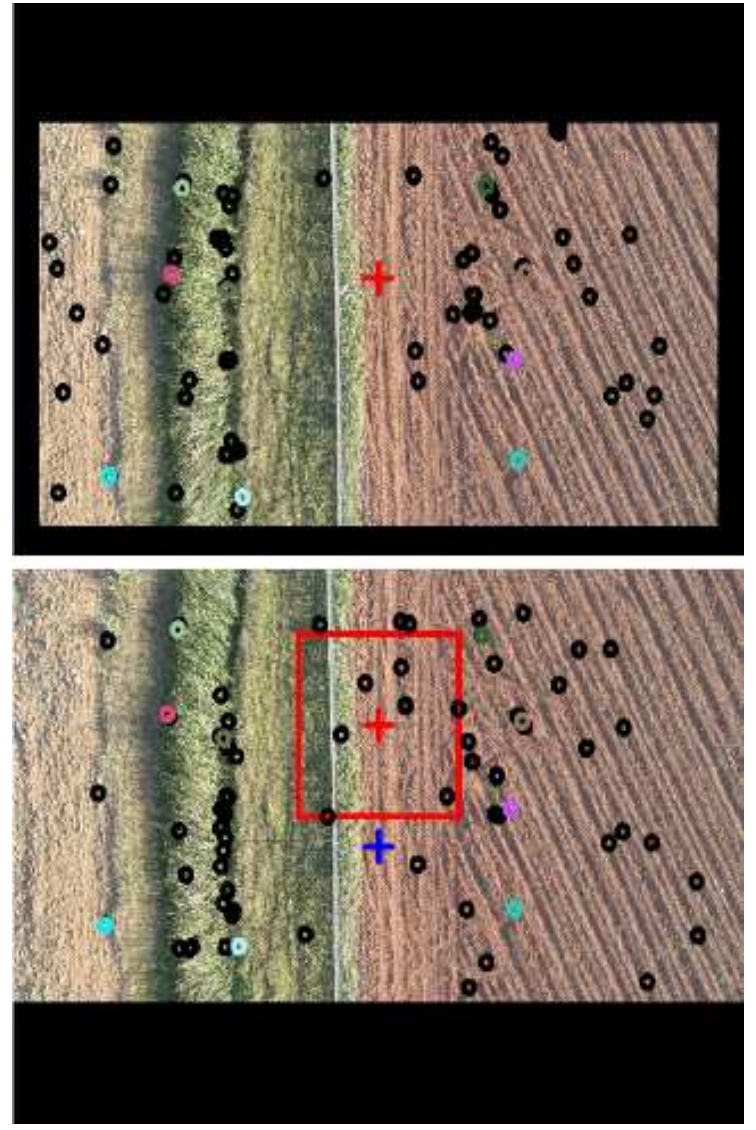

Figura 2: Asociación de características en dos imágenes tomadas en los experimentos de inspección de gasoductos.

forma $A x \approx 0$ :

$$
\left[\begin{array}{ccccc}
x_{1} & -y_{1} & 1 & 0 & -x_{2} \\
y_{1} & x_{1} & 0 & 1 & -y_{2}
\end{array}\right]\left[\begin{array}{c}
\cos \theta \\
\sin \theta \\
p_{x} \\
p_{y} \\
1
\end{array}\right]=\left[\begin{array}{l}
0 \\
0
\end{array}\right]
$$

Por lo que su solución en el sentido de los mínimos cuadrados viene dada por la expresión $A^{T} A x^{*}=$ 0 . Para su resolución, se emplea la descomposición en valores singulares (SVD). A puede ser factorizada como $A=U \Sigma V^{T}$, donde las columnas de $V$ se corresponden con los autovectores de $A^{T} A$. Las componentes de los autovectores asociados al autovalor más próximo a cero serán los parámetros que permiten el mejor ajuste. Una vez calculada la matriz $T$, se aplica la transformación a todos los puntos de la imagen.

\section{EXPERIMENTOS}

El método se ha validado experimentalmente en aplicaciones de inspección y mantenimiento de oleoductos. Los experimentos se realizaron con el robot DARIUS (véase Figura 3) desarrollado por el Laboratorio de Robótica, Visión y Control. DARIUS tiene un MTOW (Maximum Take Off Weight) de $22 \mathrm{~kg}$ y un tamaño de hélice de 1700 mm. Para estos experimentos DARIUS fue equipado con un GPS, una cámara de alta resolución orientada hacia el suelo y un sensor para medir la concentración de gases hidrocarburos y poder detectar fugas. También estaba equipado con un LiDAR Velodyne HDL-32 que no se empleó en estos experimentos.

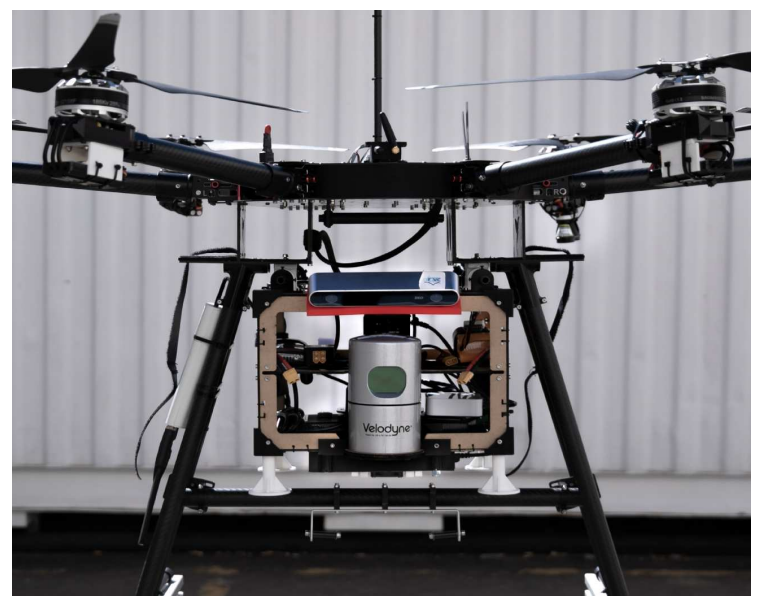

Figura 3: Robot aéreo utilizado en los experimentos.

Se realizaron una serie de vuelos con una duración media de unos 5 minutos. De media en cada uno de ellos el robot realizó un vuelo de $200 \mathrm{~m}$ de distancia. El robot sobrevoló la escena (supuesta casi plana) a una altura aproximada de $20 \mathrm{~m}$. El vuelo se realizó a altura constante. Se tomaron imágenes a una tasa aproximada de una imagen cada $2 \mathrm{~m}$. En la Figura 4 se presenta un mosaico obtenido en un pequeño recorrido del robot. Este resultado integra 15 imágenes. No se muestra el mosaico completo por motivos de espacio y resolución de la figura.

Además, el método proporciona la información recogida por el sensor de concentración y la georeferencia de cada captura como imágenes que pueden ser superpuestas sobre el mosaico. Esta información resulta de gran interés para el operario que realice la inspección, por lo que obtenerla de forma visual supone un gran beneficio.

La generación del mosaico completo ha tardado aproximadamente 45 segundos. La unión de dos imágenes se realiza en un tiempo entre 0.2 y 0.3 segundos, de los cuales aproximadamente el $70 \%$ se invierte en la extracción de características, cerca de un $10 \%$ en su asociación y el $20 \%$ restante, en obtener y aplicar la transformación.

Los parámetros de las transformaciones calculados para generar el mosaico permiten conocer la 


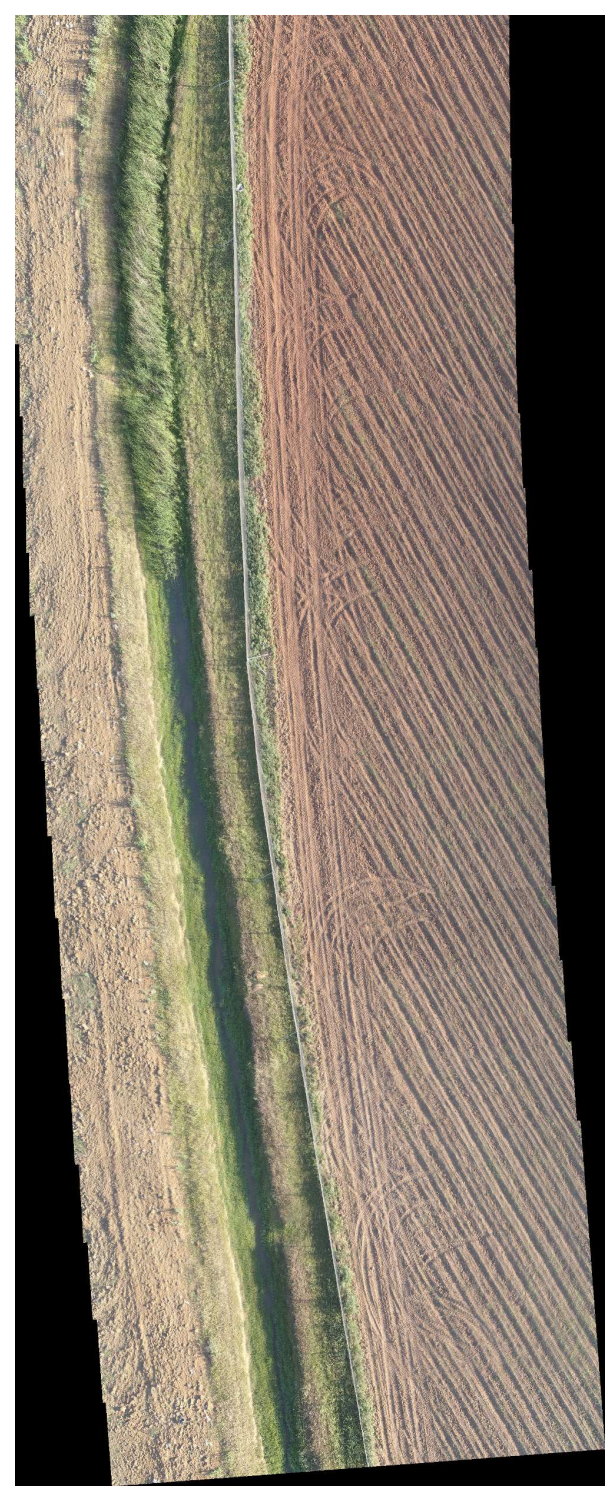

Figura 4: Mosaico generado en un tramo de recorrido.

posición y orientación del robot aéreo durante la inspección. La Figura 5 muestra la trayectoria descrita por el robot durante uno de los experimentos, en el que se integraron 50 imágenes.

Para comprobar el grado de calidad del resultado obtenido se comparó con el ofrecido por el software profesional de fotogrametría y mapeado $P i x 4 D$, el cual necesitó 4 minutos para generar el mosaico. La parte correspondiente al tramo presentado anteriormente puede observarse en Figura 6 . El método propuesto ha generado un resultado de calidad similar pero en aproximadamente un $40 \%$ del tiempo empleado por Pix4D.

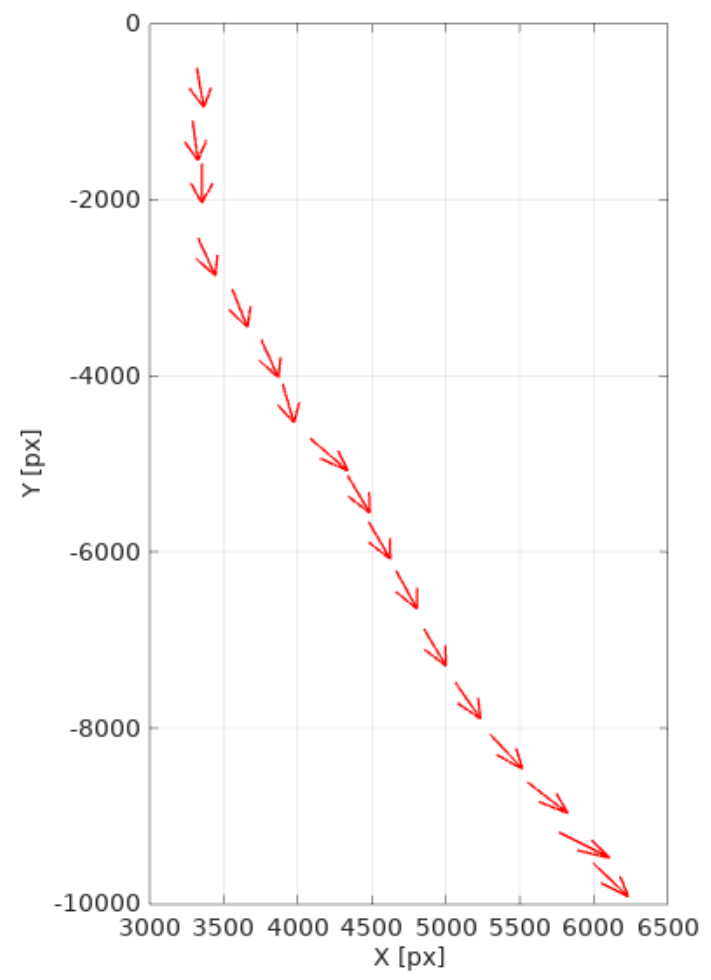

Figura 5: Posición y orientación del robot aéreo durante uno de los experimentos.

\section{CONCLUSIONES}

La generación de mosaicos de imágenes tomadas por robots aéreos tiene una amplia variedad de usos y aplicaciones. A pesar de haberse desarrollado numerosas técnicas de generación de mosaicos, los métodos del estado del arte aún tienen un elevado coste computacional que exige su implementación fuera de línea, lo que implica limitaciones en muchas aplicaciones. En este artículo estamos interesados la inspección de infraestructuras lineales, la cual requiere la generación de mosaicos de gran tamaño. Además, es necesario que los métodos operen en condiciones cercanas al tiempo real.

Este artículo presenta un método de generación de mosaicos mediante imágenes tomadas por robots aéreos para aplicaciones de inspección de infraestructuras lineales, tales como gasoductos $\mathrm{u}$ oleoductos. El método se basa en detectar puntos característicos que son asociados en imágenes consecutivas y a partir de los cuales se obtiene la matriz de transformación entre las imágenes. Sin embargo, cada una de las fases anteriores ha sido diseñada para tener un comportamiento robusto y preciso con un moderado coste computacional lo que permite su implementación en casi tiempo real. El método utiliza la hipótesis de vuelo rectilíneo de altura constante en un escenario plano y la estimación del desplazamiento entre imáge- 


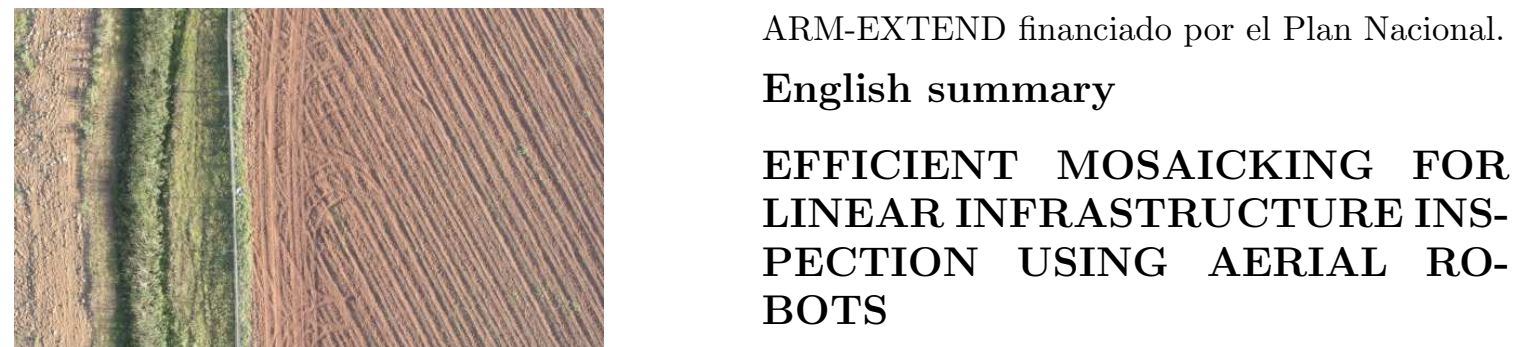

\begin{abstract}
This paper presents a mosaicking generation method using images captured by aerial robots for linear infrastructure inspection applications. The method has been designed using the problem hypotheses in order to reduce its computational cost, keeping its precise and robust performance. Particularly, it uses the rectilinear flight hypothesis and the estimation of the displacement between consecutive images to select regions of interest avoiding to detect and match features in the whole image, also reducing the outliers and, therefore, simplifying the optimization for calculating the transformation between images. The method has been validated experimentally on gas pipeline inspection missions with aerial robots.
\end{abstract}

Keywords: Aerial robotics, mosaicking, inspection.

Figura 6: Mosaico ofrecido por el software Pix $4 D$.

nes consecutivas para seleccionar zonas de interés evitando detectar y asociar características en toda la imagen, lo que redunda en menor esfuerzo computacional y menor número de valores atípicos que simplifican la optimización para el cálculo de la transformación entre imágenes. El método ha sido validado experimentalmente en misiones de inspección de oleoductos.

La relajación de la hipótesis de tierra plana es un aspecto de investigación futura. Esto permitiría al método operar en situaciones donde sea necesario la unión de imágenes con regiones a distintas alturas. Aunque el método ha sido validado experimentalmente, sería necesario extender la validación con escenarios más complejos y más extensos.

\section{Agradecimientos}

Este artículo se ha financiado por el proyecto

\section{Referencias}

[1] Aeroarms project, aeroarms-project.eu, Junio 2019.

[2] Aerobi project, aerobi.eu, Junio 2019.

[3] Aghaei, M., Leva, S. y Grimaccia, F., (2016) $\mathrm{PV}$ power plant inspection by image mosaicing techniques for IR real-time images, IEEE 43 rd Photovoltaic Specialists Conference.

[4] Bay, H., Tuytelaars, T. y Van Gool, L., (2006) SURF: Speeded Up Robust Features, European Conference on Computer Vision, pp. 404-417.

[5] Colombo, C., Del Bimbo, A. y Pernici, F., (2004) Image mosaicing from uncalibrated views of a surface of revolution, Proc. of British Machine Vision Conf. 
[6] Duplaquet, M.L., (1998) Building large image mosaics with invisible seam-lines, Proc. of the SPIE Congres AeroSence, pp. 369-377.

[7] Ghosh, D., Kaabouch, N., (2016) A survey on image mosaicing techniques, Journal of $\mathrm{Vi}$ sual Communication y Image Representation, pp. 1-11.

[8] Heikkila, M. y Pietikainen, M., (2005) An image mosaicing module for wide-area surveillance, Proceedings of the Third ACM Int. Workshop on Video Surveillance and Sensor Networks, pp. 11-18.

[9] Inampudi, R.B., (1998) Image mosaicing, International Geoscience and Remote Sensing Symposium Proc.

[10] Jethwa, M., Zisserman, A. y Fitzgibbon, A., (1998) Real-time panoramic mosaics and augmented reality, Proc. of the Britsh Machine Vision Conf., pp. 852-862.

[11] Jianchao, Y. y Chern, C.T., (2001) The practice of automatic satellite image registration, Asian Conf. on Remote Sensing, pp. 5-9.

[12] Karami, E., Prasad, S. y Shehata, M., (2017) Image matching using SIFT, SURF, BRIEF and ORB: performance comparison for distorted images, ArXiv.

[13] Kekec, T., Yildirim, A. y Unel, M., A new approach to real-time mosaicing of aerial images, Robotics and Autonomous Systems, pp. 1755-1767.

[14] Kern, A. y Bobbe, M., (2016) Towards a realtime image mosaicing solution, Int. Micro Air Vehicles Conference.

[15] Li, Y., Randall, C.J., van Woesik, R. y Ribeiro, E., (2019) Underwater video mosaicing using topology and superpixel-based pairwise stitching, Expert Systems with Applications, pp. 171-183.

[16] Lowe, D.G., (2004) Distinctive image features from scale-invariant keypoints, International Journal of Computer Vision, pp. 91-110.

[17] McLauchlan, P.F. y Jaenicke, A., (2000) Image mosaicing using sequential bundle adjustment, Proc. of the British Machine Vision Conf., pp. 616-625.

[18] Rousso, B., Peleg, S., Finci, I. y Rav-Acha, A., (1998) Universal mosaicing using pipe projection, Proc. of the Sixth IEEE Int. Conf. on Computer Vision, pp. 945-952.
[19] Rublee, E., Rabaud, V., Konolige, K., Bradski, G., (2011) ORB: an efficient alternative to SIFT or SURF, International Conference on Computer Vision.

[20] Zagrouba, E. y Barhoumi, W., (2009) An efficient image-mosaicing method based on multifeature matching, Machine Vision and Applications, pp. 139-162.

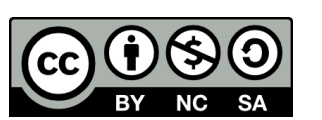

(c) 2019 by the authors. Submitted for possible open access publication under the terms and conditions of the Creative Commons Attribution CC BY-NC-SA 4.0 license (https://creativecommons.org/licenses/by-ncsa/4.0/deed.es). 